\title{
Self-focusing and spherical aberrations in corneal tissue during photodisruption by femtosecond laser
}

\author{
Valeria Nuzzo*, \\ Laboratoire d'Optique Appliquée \\ ENSTA-École Polytechnique-CNRS UMR 7639 \\ Chemin de la Hunière \\ 91761 Palaiseau cedex, France
}

\author{
Michèle Savoldelli \\ Jean-Marc Legeais \\ Hôpital Hôtel Dieu \\ Université Paris Descartes \\ Laboratoire Biotechnologie et OEi \\ EA 4063, 1 place du parvis Notre Dame \\ 75181 Paris cedex 4, France
}

\section{Karsten Plamann}

Laboratoire d'Optique Appliquée

ENSTA-École Polytechnique-CNRS UMR 7639

Chemin de la Hunière

91761 Palaiseau cedex, France

\begin{abstract}
The use of ultrashort pulse lasers is current in refractive surgery and has recently been extended to corneal grafting (keratoplasty). When performing keratoplasty, however, permanent degradation of the optical properties of the patient's cornea compromises the penetration depth of the laser and the quality of the incisions, therefore causing unwanted secondary effects. Additionally, corneal grafting needs considerably higher penetration depths than refractive surgery. Little data are available about the interaction processes of the femtosecond pulses in the volume of pathological corneas-i.e., in the presence of spherical aberrations and optical scattering. We investigate the influence of the focusing numerical aperture on the lasertissue interaction. We point out that at low numerical apertures (NAs), tissue damage is produced below and above the focal region. We attribute this phenomenon to nonlinear self-focusing effects. On the other hand, at high NAs, spherical aberrations become significant when focusing at high depths for posterior surgeries, which also limit the cutting efficiency. As high NAs are advisable for reducing unwanted nonlinear effects and ensure accurate cutting, particular attention should be paid to aberration management when developing clinical femtosecond lasers. ๑ 2010 Society of Photo-Optical Instrumentation Engineers. [DOI: 10.1117/1.3455507]
\end{abstract}

Keywords: femtosecond laser; cornea; tissue surgery; self-focusing; spherical aberrations; laser-tissue interaction.

Paper 09566R received Dec. 21, 2009; revised manuscript received Apr. 29, 2010; accepted for publication May 3, 2010; published online Jun. 30, 2010.

\section{Introduction}

Ultrashort pulse lasers represent a powerful tool for tissue disruption and cell nanosurgery, as the laser interaction process with transparent tissue is strongly localized, allows surgery in the depth of the tissue without needing access from the surface, and causes only negligible side effects. ${ }^{1}$ The first clinical application of near-infrared (NIR) femtosecond (fs) lasers in ophthalmology has been their use in refractive surgery. ${ }^{2,3}$ The most common technique for correcting ametropia is called laser in situ keratomileusis (LASIK), in which fs lasers have replaced traditional handheld surgical blades for cutting the superficial tissue flap. ${ }^{4-6}$ A sizable market has developed, and several clinical systems are now commercially available. Recently, the application of these lasers has extended to corneal transplantation or keratoplasty, ${ }^{7}$ traditionally performed using mechanical microkeratomes. Different transplantation techniques exist, which are divided into penetrating and partial lamellar keratoplasties. ${ }^{8}$ Whereas in penetrating keratoplasty, a full-thickness corneal button is replaced, partial lamellar transplantations involve the selective replacement of corneal layers. Regardless of the type of keratoplasty performed, femtosecond lasers provide precise corneal tissue cut-

*Current address: Harvard University, School of Engineering and Applied Physics.

${ }^{+}$Address all correspondence to Valeria Nuzzo, Tel: 617-495-9616; Fax: 617 495-2875; E-mail: valeria.nuzzo@polytechnique.edu ting, ensuring a regular shape of the cut edges and a better match between the donor button and the recipient tissue. ${ }^{9-12}$

The physical mechanism behind tissue photodisruption in the femtosecond regime is the laser-induced optical breakdown (reviewed in Ref. 1). Nonlinear absorption of a tightly focused laser in the bulk of an otherwise transparent tissue creates a plasma, which expands due to local elevated temperature and pressure and subsequently collapses, forming a cavitation bubble. The optical breakdown is a deterministic phenomenon. It occurs at a threshold irradiance $I_{t h}$ whose value depends on the pulse duration. For femtosecond pulses, $I_{t h}$ is on the order of $10^{13} \mathrm{~W} / \mathrm{cm}^{2}$. Previous studies report that optical breakdown and damage in dielectrics were obtained at radiant exposures of 1 to $2 \mathrm{~J} / \mathrm{cm}^{2}$ (Refs. 13 and 14). Obviously, the focusing numerical aperture is a key parameter determining the quantity of the energy needed to be delivered to the tissue for reaching threshold radiant exposure for optical breakdown. It also influences the spatial quality of the beam at the focal volume. Indeed, very fine and highly localized laser effects may be induced in the material by employing high numerical apertures (NAs). This also allows using low energies in the nanojoule range ${ }^{15}$ and prevents permanent modifications of the tissue from forming due to possible unwanted nonlinear effects generated at low NAs. However, when using high numerical apertures, spherical aberrations

$1083-3668 / 2010 / 15(3) / 038003 / 8 / \$ 25.00$ (C) 2010 SPIE 
introduced by the high focusing depth needed for posterior surgery can no longer be neglected. (Numerical apertures considered for clinical applications typically are not close to or above 1 . Although in principle conceivable, the use of immersion optics would not be practical under clinical conditions.) Moreover, the typical pathologies in the patient cornea, which requires keratoplasty, strongly alter their optical quality. The edema (increase in liquid content) typically present in these corneas modifies the otherwise very constant distances between neighboring collagen fibrils in the corneal stroma, thereby perturbing their short-distance order and reducing corneal transparency. ${ }^{16}$ Furthermore, the resulting fluctuations in the fibril density cause variations in the refractive index, resulting in additional spherical aberrations. Distortion of the point-spread function due to scattering and optical aberration influences plasma formation ${ }^{17}$ and tissue disruption accuracy. It also limits the working depth for a given pulse energy. ${ }^{18}$

In the present study, we probe the influence of the numerical aperture on the fs laser cutting of corneal tissue. Focusing the beam with low to moderate NAs for performing incisions in a lamellar plane parallel to the surface induced nonlinear effects outside the focal volume. Tight focusing reduced the extent of these effects. However, by using a numerical model, we showed that at high numerical apertures, the spherical aberrations strongly degrade the spatial quality of the beam focused into the cornea. Based on this result, we experimentally verified the possibility of correcting spherical aberrations using specific microscope objectives when inducing posteriorto-interior incisions.

\section{Experimental Methods}

\subsection{Experimental Assays and Numerical Modeling}

We performed two series of incisions in opaque corneas (prepared as described in the following subsection):

- Lamellar incisions obtained using as focusing optics an achromat doublet lens of numerical aperture (NA) of 0.15 $(f=50 \mathrm{~mm}$, Melles Griot) and three microscope objectives of $\mathrm{NA}=0.3,0.5$, and 0.75 (UPLFL, Olympus). Incisions were induced at a depth of $150 \pm 10 \mu \mathrm{m}$ in seven corneas.

- Posterior-to-anterior incisions obtained using two objectives with $\mathrm{NA}=0.4$ and 0.6 , specifically designed to correct the spherical aberration at a given focusing depths (LD PlanNeofluar, Zeiss). Six corneas were irradiated.

Due to the relative opacity of the cornea, the energy used was adjusted at a value corresponding to four times the threshold radiant exposure at the surface.

Using the ZEMAX software permitting numerical modeling of optical systems, we estimated the contribution of spherical aberrations to the decrease of the irradiance as a function of the numerical aperture at focusing depths ranging up to $1000 \mu \mathrm{m}$, which is the typical thickness of a pathological cornea.

\subsection{Setup and Sample Preparation}

We performed experiments on human corneal grafts obtained from the Banque Française des Yeux (French Eye Bank, BFY). The study was conducted according to the tenets of the Declaration of Helsinki and the French legislation for scientific use of human corneas. The corneo-scleral buttons were recovered from one to two weeks after enucleation and stored

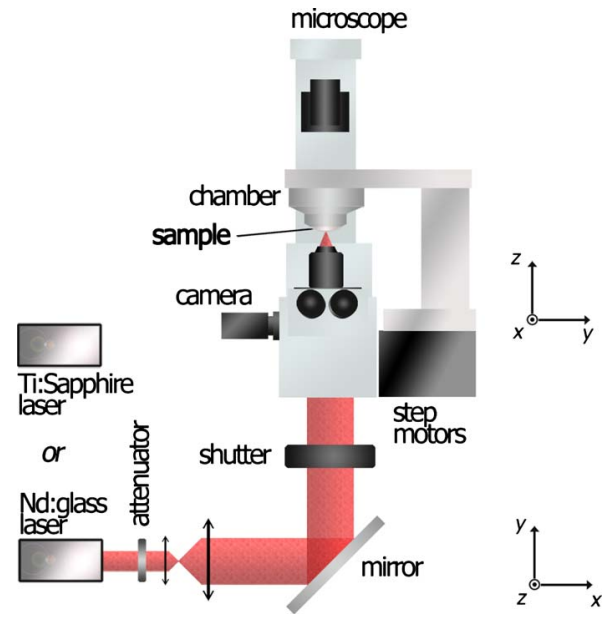

Fig. 1 Schema of the experimental set up.

in CorneaMax solution at room temperature until the experiments. The buttons made available to us for research purposes had previously been considered unsuitable for transplantation by the BFY. They generally presented a defective endothelium, which normally ensures the regulation of the corneal hydration. As a consequence, bathing them in the solution promotes swelling, thus reproducing corneal edema.

The cornea was mounted onto an artificial chamber specifically conceived to reproduce natural mechanical and pressure conditions and to maintain the natural curvature of the specimen. By filling the chamber with a bicarbonate-buffered saline (BBS) solution, a pressure is applied to the cornea and the hydration of the tissue is preserved. The sample could be positioned in three dimensions by step motors with submicrometric resolution, allowing a laser scan in three dimensions.

For cutting the corneal tissue, we used two laser systems:

- A mode-locked Ti:Sapphire oscillator (Mira, Coherent), followed by a chirped pulse amplification (CPA) system with a regenerative amplifier. The laser emits pulses with durations of about $150 \mathrm{fs}$, at a repetition rate of $1 \mathrm{kHz}$, a central wavelength of $800 \mathrm{~nm}$, and a maximum energy of $20 \mu \mathrm{J}$.

- A mode-locked diode-pumped Nd:glass oscillator, followed by CPA and regenerative amplification system, delivering pulses with duration of about $500 \mathrm{fs}$, at a repetition rate of $10 \mathrm{kHz}$, a central wavelength of $1.06 \mu \mathrm{m}$, and a maximum energy of $6 \mu \mathrm{J}$. This laser system has characteristics similar to the sources used in typical early clinical lasers.

In both configurations, a beam expander allowed overfilling the pupils of the objective, which focused the laser beam into the sample. Focusing optics were mounted either on an independent holder or onto an inverted optical microscope inserted into the beam path. In the latter case, in order to preserve the in situ configuration, a chamber in Plexiglas was used and a camera was mounted on the side of the microscope with a video port. The experimental setup is presented in Fig. 1.

Following laser cutting, the samples were prepared for histological and ultrastructural analysis by light microscopy (Zeiss Phomi2) and transmission electron microscopy (TEM; Philips CM10), respectively. They were chemically fixed and embedded in a resin. Subsequent semithin and ultrathin sec- 

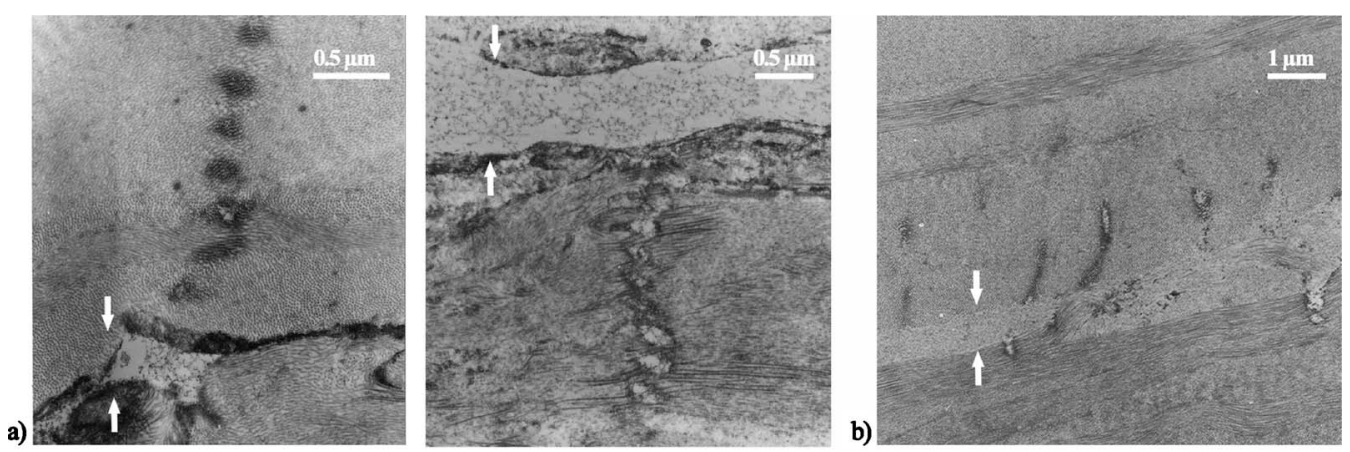

Fig. 2 TEM photographs of a corneal section showing out-of-focus modifications. Arrows show the lamellar plane parallel to the surface in which the incision was performed. (a) When focusing the laser with $N A<0.5$ for producing a lamellar cut, periodical modifications of the tissue are induced below and above the focal plane. The tissue appears darkened in the TEM photographs, suggesting photochemical modifications (left) or cut following optical breakdown (right). (b) At $0.75 \mathrm{NA}$ focusing, the modification does not show periodic features.

tions were obtained using an ultramicrotome (Reichert OmU2). Semithin sections were stained with toluidine blue, while ultrathin sections were colored by uranyl acetate and lead citrate solutions for observation.

\section{Results and Discussion}

\subsection{Laser-Induced Streaks}

Using light microscopy, we performed an a posteriori analysis of lamellar corneal incisions. Analysis showed that laserinduced side effects occurred on the optical axis well outside the point-spread function (PSF) of the focused beam. They correspond to darkening of the tissues as streaks, which form above and below the lamellar plane where the cut was obtained. This effect occurred when using focusing numerical apertures of $0.15,0.3,0.5$, and 0.75 . It was observed in all irradiated corneas, although not in all analyzed histological sections. Electron transmission microscopy of corneal ultrathin sections revealed that the permanent modifications produced in the cornea when using NAs ranging between 0.15 and 0.5 have a striking periodical structure as a consequence of laser irradiation. In some cases, the tissue is just stained (i.e., modified in a way that reduces its transparency for electrons); in others, collagen fibrils have been disrupted [Fig. 2(a)]. Lamellar incisions performed at $0.75 \mathrm{NA}$ showed no periodical features, and the extent of the out-of-focus modification is strongly reduced, reaching a maximum length of $2.0 \pm 0.2 \mu \mathrm{m}$ [Fig. 2(b)]. We measured the period from TEM photographs. The corresponding values are reported in the graph in Fig. 3, where we have classified them by the numerical aperture used. The measured average period ranges between $0.22 \pm 0.05 \mu \mathrm{m}$ and $0.47 \pm 0.09 \mu \mathrm{m}$. The periodical structure can extend up to $30 \mu \mathrm{m}$, and there is no qualitative decrease of the effect produced by the laser (staining or disruption) as we move away from the lamellar plane of the incision.

Evidence of material modification in the form of streaks has been reported in previous studies for corneal tissue; ${ }^{19}$ however, a periodical feature was not observed, probably due to analysis at a lower resolution. To the best of our knowledge, we are first to show the occurrence of self-organized features into corneal tissue.
We believe that the out-of-focus modifications induced in the tissue when performing lamellar incisions are due to local maxima in the laser intensity. This hypothesis is supported by the observation that in some corneal sections, optical breakdown occurred, thus disrupting the collagen fibrils. When the radiant exposure threshold for optical breakdown is not reached, the staining of the tissue is likely generated by lowdensity plasma oxidizing the fibrils. A previous work showed that by nonlinear absorption of NIR femtosecond pulses, photolysis of water molecules occurs producing reactive oxygen species (ROS), such as $\mathrm{OH}^{-}$and $\mathrm{H}_{2} \mathrm{O}_{2}$ (Ref. 19). In addition, cell damage induced by NIR irradiation and ascribed to photochemical effects has been previously reported. ${ }^{20}$ In our studies, periodic tissue modifications might be produced by nonlinear effects related to an increase of the intensity-dependent refractive index, causing self-focusing and subsequent plasma defocusing, which likely occur at low and moderate numerical apertures. $^{21-23}$ The combination of these two phenomena results in a successive focusing-defocusing effects, giving rise to filamentation. For ultrashort irradiation, self-focusing oc-

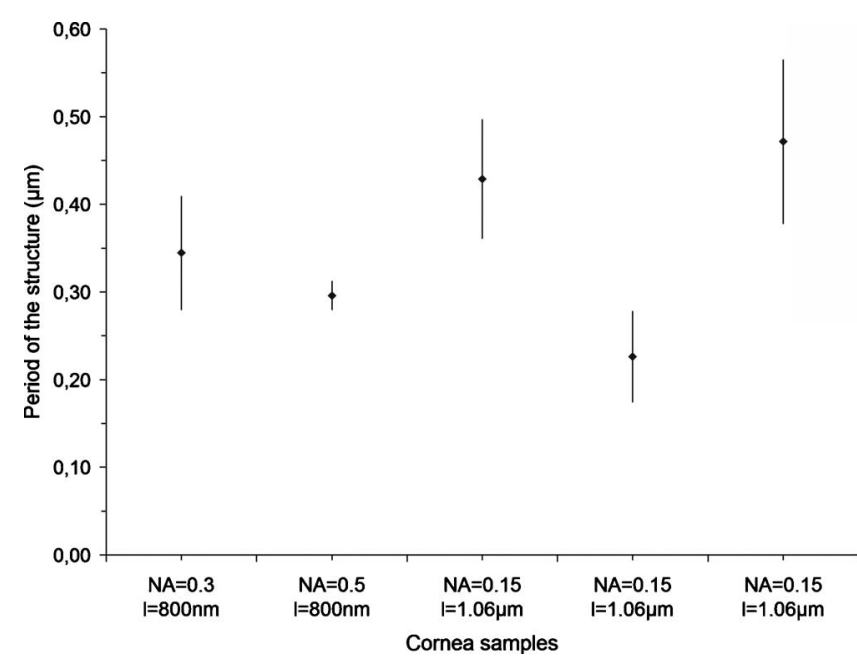

Fig. 3 Average structure period of five corneas ( \pm standard deviation) that underwent lamellar dissection. Values are classified as a function of the focusing optics NA and the laser wavelength. Periods were measured from TEM photographs. 
Table 1 Values for the experimental threshold energy (10\% measurement uncertainty), four times the power corresponding to the experimental threshold energy, and the critical power for self-focusing to be produced, as a function of different laser parameters.

\begin{tabular}{llllc}
\hline & $\begin{array}{l}\mathrm{NA}=0.15, \\
\lambda=1.6 \mu \mathrm{m}, \\
\tau=500 \mathrm{fs}\end{array}$ & $\begin{array}{l}\mathrm{NA}=0.3, \\
\tau=800 \mathrm{~nm}, \\
\tau=150 \mathrm{fs}\end{array}$ & $\begin{array}{l}\mathrm{NA}=0.5, \\
\tau=1500 \mathrm{~nm},\end{array}$ & $\begin{array}{c}\mathrm{NA}=0.75, \\
\tau=800 \mathrm{~nm}, \\
\tau=150 \mathrm{fs}\end{array}$ \\
\hline $\begin{array}{l}\text { Experimental threshold } \\
\text { energy }\left(E_{\text {texp }}\right)\end{array}$ & $2 \mu \mathrm{J}$ & $280 \mathrm{~nJ}$ & $130 \mathrm{~nJ}$ & $60 \mathrm{~nJ}$ \\
$\begin{array}{l}4 \times \mathrm{P} \\
\text { corresponding } \\
\text { to the } E_{\text {texp }}\end{array}$ & $16 \mathrm{MW}$ & $7.5 \mathrm{MW}$ & $3.4 \mathrm{MW}$ & $1.6 \mathrm{MW}$ \\
$P_{\text {cr }}$ & & & \\
\hline
\end{tabular}

curs when the pulse peak power exceeds a critical power $P_{c r}$ that in the case of a Gaussian beam is given $b^{24,25}$ :

$$
P_{c r}=3.77 \frac{\lambda^{2}}{8 \pi n_{0} n_{2}},
$$

where $n_{0}$ and $n_{2}$ stand for the linear and nonlinear refractive indexes of the material. Considering $n_{0 \text {-cornea }}=1.38$ and $n_{2 \text {-cornea }} \approx n_{2 \text {-water }}=1.2 \times 10^{-6} \mathrm{~cm}^{2} / \mathrm{W}$, using the value of water to model the nonlinear properties of corneal tissue, $P_{c r}$ corresponds to values of $10.2 \mathrm{MW}$ at $\lambda=1.06 \mu \mathrm{m}$ and $5.8 \mathrm{MW}$ at $\lambda=800 \mathrm{~nm}$. As the occurrence of self-focusing depends on laser power, whereas photodisruption is dependent on irradiance, the strength of the focusing will determine which mechanism will prevail. Because of the smaller focusing area, high NAs permit us to use lower pulse energies and to remain below the critical power. Based on our experimental conditions, we calculated the peak powers that we reached in order to induce optical breakdown. The values are reported in Table 1 . We recall that the incisions were performed at a depth of $150 \pm 10 \mu \mathrm{m}$ and at four times the surface threshold irradiance in order to ensure optical breakdown in the bulk of nontransparent samples. Although the beam experiences attenuation, which reduces the radiant exposure in the volume of the tissue, we estimate that the power is in a range where self-focusing is generated, for $\mathrm{NA}=0.15$ and $\mathrm{NA}=0.3$. In fact, based on considerations on the laser propagation in opaque corneas reported in a previous investigation, ${ }^{18}$ typically for a cornea with mild edema, the $1 / e$ penetration depth is in the order of $300 \mu \mathrm{m}$. According to Eq. (4) (see the following), the energy at $150 \mu \mathrm{m}$ is attenuated by a factor of 0.6 , which would support our estimation.

Our analysis revealed that the lateral dimensions of the features obtained typically measure a fraction of the beam waist. This is in agreement with studies on filamentation in air $^{26}$ and in other transparent media (reviewed in Ref. 25). The high degree of nonlinearity of the process and different experimental parameters may explain the discrepancy between the subwavelength values of the periodical structure when focusing into the cornea and values of multiples of the wavelength obtained in these previous studies. ${ }^{25}$

At an NA of 0.75 , the power necessary to obtain photodisruption is well below the critical power for self-focusing, and the streak could be attributed to low plasma density produced in the Rayleigh range equal to $1.6 \mu \mathrm{m}$. An NA of 0.5 seems to correspond to a transitional regime for self-focusing to occur. At lower numerical apertures, other mechanisms are unlikely to explain the observed laser effect. Constructive interferences between the incident beam and the beam reflected by the plasma created in the focal plane cannot account for the effects observed below the focal plane (considering that the plasma is not reflecting in the forward direction). It does not seem probable either that local maxima in the axial light distribution in the focal volume (assumed to be an Airy pattern in our experiments) may cause the observed patterns. The first maxima of intensity of the Bessel function describing the irradiance distribution is a distance of $130 \lambda(\mathrm{NA}=0.15)$, $30 \lambda(\mathrm{NA}=0.3)$, and $9 \lambda(\mathrm{NA}=0.5)$ from the focal plane, which is much greater than the period lengths we measured. Moreover, secondary maxima being less intense, an attenuation of the laser effects should be visible with increasing distance from the focal plane, which is in contrast with our results. Also, nanostructures have been reported in silicon attributed to nonlinear nanoplasmonic processes; however, the periodicity is produced in a plane parallel to the surface of the sample and the scanning plane ${ }^{27}$ and not in the incident plane, as in our case.

\subsection{Influence of Spherical Aberrations on the Laser Focusing}

According to previous studies (reviewed in Ref. 1) and in agreement with our experimental results presented earlier, increasing the focusing numerical aperture can reduce the extent of out-of-focus material modifications caused by nonlinear effects. However, at high NAs, spherical aberrations may become significant-in particular, when cutting opaque tissue-as they contribute to the broadening of the pointspread function and therefore raise the energy threshold and reduce the laser accuracy. In order to evaluate the beam focus degradation ascribed to spherical aberrations at different NAs as a function of the focusing depth, we performed numerical simulations using the ZEMAX software. Based on geometric ray tracing, ZEMAX allows the analysis of modeled optical systems. In particular, we computed wavefront aberrations in order to determine the Strehl ratio at different focusing depths. For our simple optical system consisting of optics 


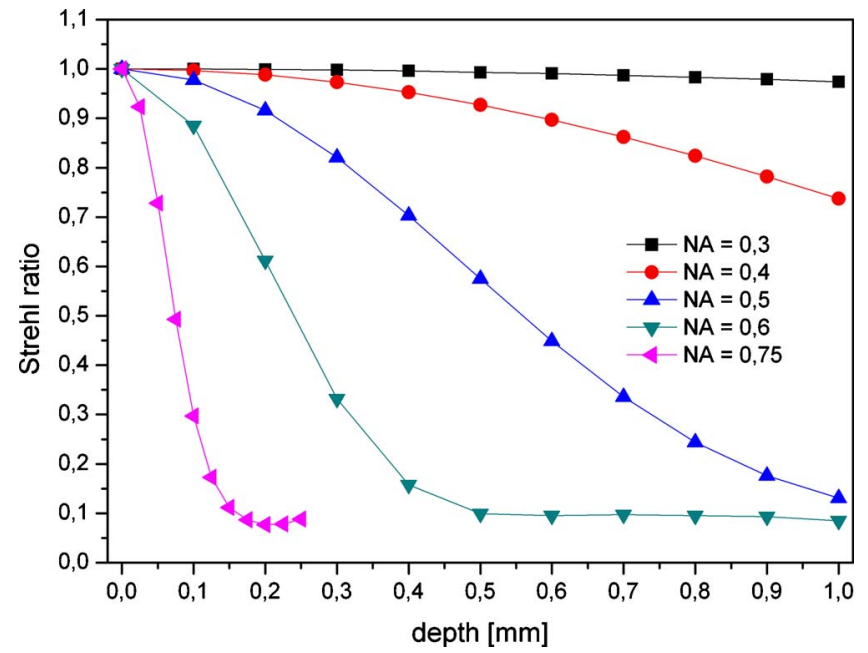

Fig. 4 Variation of the Strehl ratio at different numerical apertures as a function of the focusing depth into the cornea. Values are obtained using the RMS method.

focusing the beam into the cornea, the geometric approximation appears to be adequate. Design data for the doublet lens were available in the ZEMAX catalogue. The microscope objectives were modeled by a paraxial element corresponding to their numerical aperture, as we assumed that their pointspread function is reasonably close to the diffraction limit. The cornea was simulated as an isotropic material with a refractive index of 1.37. The merit function was minimized by optimizing the wavefront at $\lambda=1.06 \mu \mathrm{m}$, which is the typical operating wavelength of clinical systems, and the beam intensity throughout the entrance pupil of the system was considered uniform. ZEMAX enables calculating the peak-to-valley $(\mathrm{P}-\mathrm{V})$ and the root-mean-square (RMS) wavefront aberrations as well as the Strehl ratio. Peak-to-valley aberration refers to the maximum wavefront departure from the ideal value in both positive and negative directions, whereas the RMS wavefront error expresses the deviation averaged over the entire wavefront. According to the literature, the Strehl ratio $S$ can be approximated by the following relation: ${ }^{28,29}$

$$
S \approx 1-(2 \pi \sigma)^{2}
$$

where $\sigma$ is the RMS value of the wavefront error. As Eq. (2) is no longer valid for Strehl ratios $<0.5$, a different approximation is used by ZEMAX to calculate the Strehl ratio. It is given by

$$
S \approx \exp \left[-(2 \pi \sigma)^{2}\right] \approx 1-(2 \pi \sigma)^{2}+\frac{(2 \pi \sigma)^{4}}{2 !}+\ldots,
$$

and is valid for Strehl ratios as small as 0.10 (Ref. 30). Figure 4 shows the Strehl ratio computed with the RMS approach as a function of the focal plane depth into the sample. The $1 / e$ penetration depths measured from the Strehl ratio curves and peak-to-valley and RMS wavefront aberrations calculated at a depth of $500 \mu \mathrm{m}$ are presented in Table 2 . We point out that these values underestimate the total aberration that the beam experiences during propagation: only spherical aberrations introduced by the working depth are considered here, and aberrations introduced by irregularities on the surface and local or low-frequency variations in the refractive index within the tissue are not accounted for.

One can immediately notice that for low numerical apertures, spherical aberrations can be neglected. Considering a maximum thickness of about $1 \mathrm{~mm}$ for an edematous cornea, the contribution to the beam attenuation becomes significant for NA $>0.5$. Using specific microscope objectives with numerical apertures of 0.4 and 0.6 , we performed posterior-toanterior incisions by correcting the spherical aberrations at a given focusing depth in the volume of the cornea. The correction depth and the yielded incision lengths, measured by light microscopy, are reported in Table 3. It can be noticed that increasing the correction depth increases the incision length up to a point beyond which this tendency is reversed. As expected, the behavior is more prominent when using the 0.6 NA focusing optics, as compared to $0.4 \mathrm{NA}$, which produces reduced spherical aberrations. We believe that as we correct deeper in the edematous tissue, the scattering becomes significant, and when associated to the degradation of the beam away from the correction point, it prevents us from cutting farther. As a result, the maximum length of a cut can be less than the total thickness of pathological corneas.

Even though the irradiance attenuation by spherical aberration does not follow an exponential behavior, for simplicity, we here associate a $1 / e$ penetration depth with this phenomenon and make a semiquantitative estimation. Therefore, if we consider that to a first approximation, both the extinction of the irradiance $I(z)$ by scattering and spherical aberrations may be described by an exponential decay, we can write

$$
I(z) \approx I_{0} \exp \left(-\frac{z}{l}\right)=I_{0} \exp \left(-\frac{z}{l_{s c}}-\frac{\left|z-z_{0}\right|}{l_{s a}}\right),
$$

where $I_{0}$, represents the incident irradiance, $z_{0}$ the depth for which the spherical aberrations have been corrected, and $l_{c s}$

Table 2 1/e penetration depths measured form the Strehl ratio curves and peak-to-valley and RMS wavefront aberrations calculated at a depth of $500 \mu \mathrm{m}$ as a function of the numerical aperture.

\begin{tabular}{llllll}
\hline Numerical aperture & 0.3 & 0.4 & 0.5 & 0.6 & 0.75 \\
\hline 1 /e penetration depth $(\mu \mathrm{m})$ & $>1000$ & $>1000$ & 620 & 300 & 90 \\
Peak-to-valley wavefront aberration & $0.048 \lambda$ & $0.164 \lambda$ & $0.450 \lambda$ & $1.088 \lambda$ & $3.776 \lambda$ \\
RMS wavefront aberration & $0.014 \lambda$ & $0.047 \lambda$ & $0.127 \lambda$ & $0.298 \lambda$ & $0.953 \lambda$ \\
\hline
\end{tabular}


Table 3 Incision lengths obtained when focusing the beam into a cornea using microscope objectives with correction of spherical aberrations at a given depth.

\begin{tabular}{|c|c|c|c|c|c|}
\hline \multirow[t]{5}{*}{$\mathbf{N A}=0.6$} & \multirow{2}{*}{$\begin{array}{c}\text { Correction depth }(\mu \mathrm{m}) \\
0\end{array}$} & \multicolumn{4}{|c|}{$\begin{array}{l}\text { Yielded incision length }(\mu \mathrm{m}) \\
\text { (4 corneas) }\end{array}$} \\
\hline & & 265 & 250 & 490 & 490 \\
\hline & 300 & 395 & 295 & 550 & 540 \\
\hline & 450 & 470 & 320 & 375 & 360 \\
\hline & 640 & 425 & 220 & 252 & 250 \\
\hline \multirow[t]{5}{*}{ NA $=0.4$} & Correction depth $(\mu \mathrm{m})$ & \multicolumn{4}{|c|}{$\begin{array}{l}\text { Yielded incision length }(\mu \mathrm{m}) \\
(2 \text { corneas })\end{array}$} \\
\hline & 0 & 425 & 445 & & \\
\hline & 200 & 470 & 475 & & \\
\hline & 400 & 435 & 475 & & \\
\hline & 600 & 360 & 390 & & \\
\hline
\end{tabular}

and $l_{s a}$ the penetration depths for attenuation due to scattering and spherical aberrations, respectively. As the optical breakdown occurs for values of the radiant exposure higher than the threshold, relation (4) becomes

$$
\frac{z}{l_{s c}}+\frac{\left|z-z_{0}\right|}{l_{s a}}<\ln \left(\frac{I_{0}}{I_{t h}}\right),
$$

which is equivalent to

$$
\begin{gathered}
z<z_{0}, \quad z<\frac{\ln \left(I_{0} / I_{t h}\right) l_{s a}+z_{0}}{\left(l_{s a} / l_{s c}\right)+1}, \quad z>z_{0}, \\
z<\frac{\ln \left(I_{0} / I_{t h}\right) l_{s a}-z_{0}}{\left(l_{s a} / l_{s c}\right)+1} .
\end{gathered}
$$

We have represented the spherical aberration penetration depth as function of the correction length, considering two sets of parameters:

- In Fig. 5(a), a beam line is plotted varying $l_{s a}$ from $300 \mu \mathrm{m}$ to $700 \mu \mathrm{m} \quad\left(100-\mu \mathrm{m}\right.$ step), with $l_{s c}$ fixed at $350 \mu \mathrm{m}$, which well represents a typical edematous cornea.

- In Fig. 5(b), a beam line is plotted varying $l_{s c}$ from $250 \mu \mathrm{m}$ to $450 \mu \mathrm{m} \quad\left(100-\mu \mathrm{m}\right.$ step), with $l_{s a}$ fixed at $620 \mu \mathrm{m}$, which according to the previous calculation corresponds to a numerical aperture of 0.5 .

In both cases

- Data are plotted for $I_{0} / I_{t h}=4$, considering edematous tissue.

- The correction lengths have been multiplied by a factor
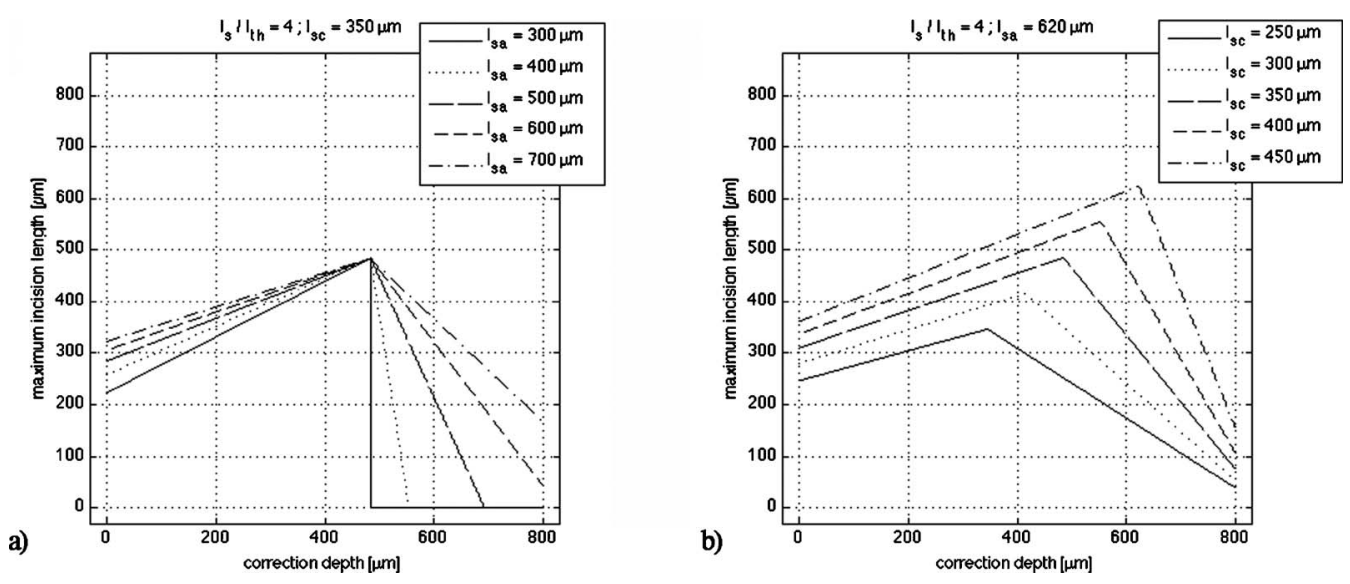

Fig. 5 Maximum incision length as a function of the correction depth for spherical aberrations. (a) $I_{s c}=350 \mu \mathrm{m}$. $I_{s a}$ varies from 300 to $700 \mu \mathrm{m}$. (b) $I_{s a}=620 \mu \mathrm{m} . I_{s c}$ varies from 250 to $450 \mu \mathrm{m}$. 
$n_{\text {glass }} / n_{\text {cornea }}=1.45 / 1.37=1.06$ as objectives are optimized for correction into glass.

- The maximum depth that can be corrected with respect to the spherical aberrations is on the line $z=z_{\max }=z_{0}$. (Otherwise, we would be able to correct for a higher depth in order to cut farther into the cornea.)

We notice that the predicted theoretical behavior is in agreement with the experimental findings: by increasing the correction depth, at a fixed set of values for $l_{s c}, l_{s a}$, and $I_{0}$, the incision length increases up to a maximum point, and then it decreases. This tendency is enhanced when $l_{s a}$ decreases, even though the variation of $l_{s a}$ does not influence the maximum incision length. At a fixed value of $l_{s a}$, the maximum incision length obviously increases with increasing $l_{s c}$.

The preceding results provide direct evidence that in pathological corneas, both scattering and spherical aberrations are limiting factors.

\section{Conclusions}

In our study, we investigated the effect of varying the focusing numerical apertures from 0.15 to 0.75 during femtosecond laser surgery of corneal tissue. We have shown that when using numerical apertures up to 0.5 , strong nonlinear effects beyond the desired optical breakdown in the focal region can occur, leading to permanent modifications of the tissue. Periodical staining or disruption features were produced in tissue below and above the focal plane when performing lamellar incision. Given the biological nature of the sample, the periodicity of the structure is remarkable, and numerical simulations merit being performed in order to investigate the nonlinear laser-tissue interaction, as well as the nonlinear propagation of the beam into the cornea.

At $0.75 \mathrm{NA}$, the extent of the modifications was strongly reduced, suggesting that higher numerical apertures should be used. Nevertheless, because in surgical applications dry objectives are typically employed for practical reasons (limiting the maximum NA to well below 1), spherical aberrations become significant, strongly reducing the spatial quality of the beam at the focal point and therefore the laser accuracy. In particular, for NA $>0.5$, the Strehl ratio of the beam decreases rapidly when increasing the NA and the beam deforms at focusing depths lower than the thickness of a pathological, as well as of a healthy, cornea.

Clinical fs laser systems for keratoplasty operate at numerical apertures typically ranging from 0.3 to 0.5 . When performing deep lamellar incisions in pathological corneal tissue, the limitations due to the aforementioned phenomena often lead to incomplete cuts. This requires the surgeon to finish the cut with a mechanical microkeratome or using a doublepass laser procedure, which reduces the interest of employing a femtosecond laser. According to our study, loosely focusing the beam produces unwanted modifications of the tissue. On the other hand, using numerical apertures from 0.5 on produces nonnegligible spherical aberration at high focusing depths. Our evaluation did not account for the additional aberrations due to the modified optical properties of a pathological cornea, which may further compromise the beam quality. We therefore believe that spherical aberrations contribute to diminishing the cutting performances of femtosecond lasers. Integrating an adaptive optics in the system would correct the beam wavefront when using high numerical apertures, which are advised for producing reduced nonlinear effects and accurate tissue cuts.

\section{References}

1. A. Vogel, J. Noack, G. Hüttman, and G. Paltauf, "Mechanisms of femtosecond laser nanosurgery of cells and tissues," Appl. Phys. B 81(8), 1015-1047 (2005).

2. T. Juhasz, F. H. Frieder, R. M. Kurtz, C. Horvath, J. F. Bille, and G. Mourou, "Corneal refractive surgery with femtosecond lasers," IEEE J. Sel. Top. Quantum Electron. 5(4), 902-910 (1999).

3. H. Lubatschowski, G. Maatz, A. Heisterkamp, U. Hetzel, W. Drommer, H. Welling, and W. Ertmer, "Application of ultrashort laser pulses for intrastromal refractive surgery," Graefe's Arch. Clin. Exp. Ophthalmol. 238(1), 33-39 (2000).

4. T. Lim, S. Yang, M. Kim, and H. Tchah, "Comparison of the IntraLase femtosecond laser and mechanical microkeratome for laser in situ keratomileusis," Am. J. Ophthalmol. 141(5), 833-839 (2006).

5. G. Sutton and C. Hodge, "Accuracy and precision of LASIK flap thickness using the IntraLase femtosecond laser in 1000 consecutive cases," J. Refract. Surg. 24(8), 802-806 (2008).

6. A. Chan, J. Ou, and E. E. Manche, "Comparison of the femtosecond laser and mechanical keratome for laser in situ keratomileusis," Arch. Ophthalmol. (Chicago) 126(11), 1484-1490 (2008).

7. K. Plamann, F. Aptel, C. Arnold, A. Courjaud, C. Crotti, F. Deloison, F. Druon, P. Georges, M. Hanna, J.-M. Legeais, F. Morin, E. Mottay, V. Nuzzo, D. A. Peyrot, and M. Savoldelli, "Ultrashort pulse laser surgery of the cornea and the sclera," J. Opt. (in press).

8. K. McColgan, "Corneal transplant surgery," J. Perioper. Pract. 19(2), 51-54 (2009).

9. F. W. Price Jr. and M. O. Price, "Femtosecond laser shaped penetrating keratoplasty: one-year results utilizing a top-hat configuration," Am. J. Ophthalmol. 145(2), 210-214 (2008).

10. L. Mosca, R. Fasciani, C. Tamburelli, L. Buzzonetti, L. Guccione, E. Mandarà, and E. Balestrazzi, "Femtosecond laser-assisted lamellar keratoplasty: early results," Cornea 27(6), 668-672 (2008).

11. J. S. Mehta, R. Shilbayeh, Y. M. Por, H. Cajucom-Uy, R. W. Beuerman, and D. T. Tan, "Femtosecond laser creation of donor cornea buttons for Descemet-stripping endothelial keratoplasty," J. Cataract Refractive Surg. 34(11), 1970-1975 (2008).

12. V. Nuzzo, F. Aptel, M. Savoldelli, K. Plamann, D. Peyrot, F. Deloison, D. Donate, and J.-M. Legeais, "Histologic and ultrastructural characterization of corneal femtosecond laser trephination," Cornea 28(8), 908-913 (2009).

13. B. C. Stuart, M. D. Feit, S. Herman, A. M. Rubenchik, B. W. Shore, and M. D. Perry, "Nanosecond-to-femtosecond laser-induced breakdown in dielectrics," Phys. Rev. B 53(2), 1749-1761 (1996).

14. D. Giguère, G. Olivié, F. Vidal, S. Toetsch, G. Girard, T. Ozaki, J. C. Kieffer, O. Nada, and I. Brunette, "Laser ablation threshold dependence on pulse duration for fused silica and corneal tissue: experiments and modelling," J. Opt. Soc. Am. 24, 1562-1568 (2007).

15. K. König, I. Riemann, P. Fischer, and K.-J. Halbhuber, "Intracellular nanosurgery with near infrared femtosecond laser pulses," Cell. Molecular Biol. 45(2), 195-201 (1999).

16. H. F. Edelhauser, "The balance between corneal transparency and edema: the Proctor lecture," Invest. Ophthalmol. Visual Sci. 47(5), 1755-1767 (2006).

17. A. Vogel, K. Nahen, D. Theisen, R. Birngruber, R. J. Thomas, and B. A. Rockwell, "Influence of optical aberrations on laser-induced plasma formation in water and their consequences for intraocular photodisruption," Appl. Opt. 38(16), 3636-3643 (1999).

18. V. Nuzzo, K. Plamann, M. Savoldelli, M. Merano, D. Donate, O. Albert, P. F. Gardeazábal Rodríguez, G. Mourou, and J. M. Legeais, "In situ monitoring of second harmonic generation in human corneas to compensate for femtosecond laser pulse attenuation in keratoplasty," J. Biomed. Opt. 12(6), 064032 (2007).

19. A. Heisterkamp, T. Ripken, T. Mamom, W. Drommer, H. Welling, W. Ertmer, and H. Lubatschowski, "Nonlinear side effects of fs pulses inside corneal tissue during photodisruption," Appl. Phys. B 74(4-5), 419-425 (2002)

20. U. K. Tirlapur, K. König, C. Peuckert, R. Krieg, and K.-J. Halbhuber, "Femtosecond near-infrared laser pulses elicit generation of reactive oxygen species in mammalian cells leading to apoptosis-like death," Exp. Cell Res. 263(1), 88-97 (2001). 
21. S. Tzortzakis, B. Lamouroux, A. Chiron, M. Franco, B. Prade, A. Mysyrowicz, and S. D. Moustaizis, "Nonlinear propagation of subpicosecond ultraviolet laser pulses in air," Opt. Lett. 25(17), 1270-1272 (2000).

22. K. Yamada, W. Watanabe, T. Toma, K. Itoh, and J. Nishii, "In situ observation of photoinduced refractive-index changes in filaments formed in glasses by femtosecond laser pulses," Opt. Lett. 26(1), 19-21 (2001).

23. L. Sudrie, A. Couairon, M. Franco, B. Lamouroux, B. Prade, S. Tzortzakis, and A. Mysyrowicz, "Femtosecond laser-induced damage and filamentary propagation in fused silica," Phys. Rev. Lett. 89(18), 186601 (2002)

24. J. H. Marburger, "Self-focusing: theory," Prog. Quantum Electron. 4, 35-110 (1975)

25. A. Couairon and A. Mysyrowicz, "Femtosecond filamentation in transparent media," Phys. Rep. 441(2-4), 47-189 (2007).

26. B. La Fontaine, F. Vidal, Z. Jiang, C. Y. Chien, D. Comtois, A.
Desparois, T. W. Johnston, J.-C. Kieffer, H. Pépin, and H. P. Mercure, "Filamentation of ultrashort pulse laser beams resulting from their propagation over long distances in air," Phys. Plasmas 6(5), 16151621 (1999).

27. V. R. Bhardwaj, E. Simova, P. P. Rajeev, C. Hnatovsky, R. S. Taylor, D. M. Rayner, and P. B. Corkum, "Optically produced arrays of planar nanostructures inside fused silica," Phys. Rev. Lett. 96(5), 057404 (2006).

28. J. C. Wyant and K. Creath, "Basic wavefront aberration theory for optical metrology," chapter 1 in Applied Optics and Optical Engineering, vol. 11, R. R. Shannon, J. C. Wyant, Eds., pp. 1-53, Academic Press, New York (1992).

29. M. Born and E. Wolf, "The diffraction theory of aberrations," chapter 9 in Principles of Optics, 7th ed., pp, 517-533, Cambridge University Press, Cambridge, UK (1999).

30. V. N. Mahajan, "Aberrated point-spread functions for rotationally symmetric aberrations," Appl. Opt. 22(19), 3035-3041 (1983). 